\title{
Sodium Valproate and Levetiracetam Treatment in Children: Their Effects on Serum Paraoxonase/ Arylesterase Activities
}

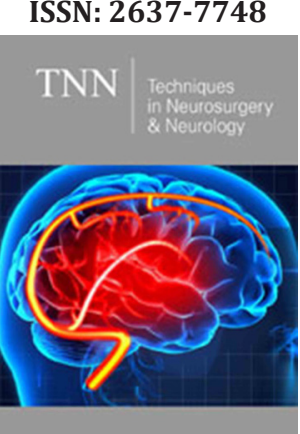

*Corresponding author: Esra Türe, Medical Doctor, Department of Pediatric Emergency, Meram Medical Faculty, Turkey

Submission: 望 February 19, 2020

Published: 侮February 25, 2020

Volume 3 - Issue 3

How to cite this article: Esra Türe, Abdullah Yazar. Sodium Valproate and Levetiracetam Treatment in Children: Their Effects on Serum Paraoxonase/ Arylesterase Activities. Tech Neurosurg Neurol.3(3). TNN.000561.2020.

DOI: 10.31031/TNN.2020.03.000561

Copyright@ Esra Türe, This article is distributed under the terms of the Creative Commons Attribution 4.0 International License, which permits unrestricted use and redistribution provided that the original author and source are credited.

\author{
Esra Türe $^{1 *}$ and Abdullah Yazar $^{2}$ \\ ${ }^{1}$ Medical Doctor, Department of Pediatric Emergency, Turkey \\ ${ }^{2}$ Associate Professor, Department of Pediatric Emergency, Turkey
}

\section{Abstract}

Objectives: Investigation of effects of seizures and used antiepileptic drugs on serum Paraoxons 1 (PON1) and aryl esterase (PONAryl) activities in patients diagnosed with epilepsy and simple febrile convulsion was aimed.

Materials and methods: Of the patients who were being followed-up with diagnosis of epilepsy and admitted to emergency department with complaint of seizure; patients using sodium valproate, levetiracetam and diagnosed with simple febrile convulsion were included in the study. In order to measure levels of PON1 and PONAryl, venous blood samples were obtained.

Results: When serum PON1 and PONAryl levels of the groups were compared, PON1 and PONAryl levels were determined to be statistically significantly lower in the epilepsy group using sodium valproate $(\mathrm{p}=0.001, \mathrm{p}=0.001)$.

Conclusion: Valproate had a potent inhibition effect and levetiracetam had weak inhibition effect on PON1 and PONAryl activities. Results suggest need for close follow-up of these patients with impaired oxidative balance due to increased risk of atherosclerosis.

Keywords: Paraoxonase; Arylesterase; Antiepileptic drugs; Epilepsy; Febrile convulsion

\section{Introduction}

Epilepsy is the most commonly seen chronic neurological disorder of the childhood, whose underlying pathophysiological mechanisms have not been well-understood yet, which is categorized in a broad spectrum as structural, metabolic, genetic, immune, infectious and of unknown etiology and affects $0.5-1 \%$ of the children [1,2]. Febrile convulsion, however, is described as a type of seizure accompanied by fever in children between six months and five years of age, without presence of any central nervous system disease or electrolyte imbalance [3]. There is a balance between free radical production and antioxidant defense system in the organism. Oxidative damage occurs when this balance is impaired on behalf of free radical production [4]. Brain tissue is extremely susceptible to lipid peroxidation, as it contains high proportion of fat and has high oxygen consumption [5]. Previous studies have shown that oxidative stress has a role in pathophysiology of epilepsy [6]. Furthermore, in the literature, there is a great number of studies regarding effect of antiepileptic drugs on oxidant or antioxidant enzymes [7]. Simple febrile seizures are generally benign but use of high amounts of glutamate and high oxygen consumption due to increased metabolic activity of the brain during seizure lead to oxidative stress [8]. Due to the thought that Paraoxonase 1 (PON1) and arylesterase (PONAryl) not only have the ability to hydrolyze organophosphate compounds and aromatic carboxylic esters, but also protects high-density lipoprotein (HDL) and lowdensity lipoprotein (LDL) from lipid peroxidation, its antioxidant effects have also been highlighted. It has been suggested that this effect is associated with peroxidase-like activity of PON1 on peroxides and with ability of PON1 to modify proportion of oxidation products within the oxidized LDL [9]. Decreased activity of PON-1 enzyme has also been reported to be a risk factor for development of atherosclerosis [10]. In this study, investigation of effects of seizures and used antiepileptic drugs on serum PON1 and PONAryl activities in patients diagnosed with epilepsy and simple febrile convulsion was aimed. 


\section{Materials and Methods}

This case control study was conducted with patients underage of 18 years who admitted to Pediatric Emergency Department of Necmettin Erbakan University Meram Faculty of Medicine with complaint of seizure between the dates January 2018 and April 2018. 30 patients using sodium Valproate and 30 patients using levetiracetam, who were being followed-up due to diagnosis of epilepsy, and 30 patients diagnosed with simple febrile convulsion were included in the study; the study was concluded after reaching to the target number of patients. In the control group, 30 healthy children age- and gender-matched for collection of blood for any reason who admitted to Pediatric Outpatient Clinics for routine pediatric follow-up, who had no septicemia and pulmonary, metabolic, rheumatological or any other disease findings were included. Informed consents were obtained from parents of both patients and the control group and the study was approved by the ethics committee of our institution. Children with mental-motor retardation, liver, kidney, cardiac or any organ system dysfunction, those receiving long term drug therapy for treatment of any disease and those who had received multivitamins within last 4 months, patients with abnormal lipid profile, obese patients, newlydiagnosed epilepsy patients and epilepsy patients who were not using any drug, those with a history of drug use for a duration shorter than six months, those receiving multiple antiepileptic drugs, those with a seizure lasting longer than five minutes and those with a family history of hypertension, diabetes mellitus, coronary artery disease, cerebrovascular disease, peripheral artery disease, heart failure, renal failure, liver failure and collagen vascular disease were not included in the study. Patients' decimal age, gender, history of drug use, age of diagnosis and types of seizures were recorded on the standard data entry form. The patients were divided into four groups (epilepsy patients using Sodium valproate, epilepsy patients using levetiracetam, those diagnosed with simple febrile convulsion and healthy controls). Epilepsy patients were compared between each other by the drugs they used, with patients with simple febrile convulsion and the control group, and the patients with febrile convulsion were compared with epilepsy patients and the control group. In order to measure levels of PON1 and PONAryl, venous blood samples were obtained from the patients (during postictal period) and the control group. The samples were centrifuged at a rate of 3000rpm for 15 minutes in order to separate serum and then kept at $-80^{\circ} \mathrm{C}$ until being analyzed. After all samples were completed, the frozen serums were melted at room temperature and examined at the same day. The activity of ARES was measured using an ARES assay kit, and the activity of PON1 was measured using a fully automated PON activity measurement kit (Rel Assay Diagnostics, Gaziantep, Turkey). The results were compared between each other and with the healthy age- and gender-matched control group. Statistical analysis of the study was performed by using Statistical Package for the Social Sciences for Windows ver. 20.0 package program. Descriptive analyses were used for analyses of distribution and frequency of the data, ChiSquare test was used for comparison of two independent groups in frequency data. For comparison of mean of two independent groups, independent samples t-test was used. For comparison of mean of more than two independent groups, however, one-way variance analysis was used. Normality analysis was performed for continuous variables. Pearson correlation analysis was used for data exhibiting normal distribution, Spearman correlation analysis was used for data which did not exhibit. For all statistical analyses, the level of statistical significance was $<0.05$.

\section{Results}

During the study period, 205 patients admitted to the pediatric emergency department because of epilepsy and 106 patients admitted because of febrile convulsion; 221 of these were not included in the study in accordance with the exclusion criteria. Of the patients; 48 (53.3\%) were female and 42 (46.7\%) were male, and mean age of all patients was $3,76 \pm 0,82$. Of the control group; 16 (53.3\%) were female and 14 (46.7\%) were male, and the mean age was $3,78 \pm 0,83$. Demographical features of the patients and the control group are represented in Table 1. No statistically significant difference was determined between patients' diagnoses and drugs and their genders ( $p>0,05)$. When duration of drug use of 60 patients using drugs was examined, it was observed that 28 (46.7\%) patients used drugs most commonly for 12-24 months. When types of seizures were examined, it was observed that 63 (\%70) patients had generalized and 27 (\%30) patients had focal seizures. No statistically significant difference was determined between patients' types of seizure and serum PON1 and PONAryl levels ( $p>0,05$ ). In correlation tests, no correlation was determined between serum PON1 and PONAryl levels and age, duration of drug use and seizure frequency ( $p>0,05)$. When mean PON1 and PONAryl levels of 90 patients who had a seizure and the healthy control group were compared, mean PON1 and PONAryl were determined to be statistically significantly lower in the patient group ( $\mathrm{p}=0,001, \mathrm{p}=0,001$ ) (Table 2). When mean serum PON1 levels of the four groups (epilepsy patients using Sodium valproate, epilepsy patients using levetiracetam, those diagnosed with simple febrile convulsion and healthy controls) were compared, mean PON1 levels was determined to be statistically significantly lower in the epilepsy group using sodium valproate $(p=0,001)$. PONAryl levels also were determined to statistically significantly lower in the epilepsy group using sodium valproate $(\mathrm{p}=0,001)$ (Table 3 ). When mean serum PON1 levels of patients using levetiracetam and healthy controls were compared, PON1 levels were determined to be statistically significantly lower in those using levetiracetam $(p=0,047)$. Serum PONAryl levels also were determined to be statistically significantly lower in those using levetiracetam compared to the healthy controls $(\mathrm{p}=0,003)$. When mean serum PON1 levels of the patients using sodium valproate and healthy controls were compared, PON1 levels were determined to be statistically significantly lower in those using sodium valproate $(p=0,001)$. Serum PONAryl levels also were determined to be statistically significantly lower in those using sodium valproate compared to the healthy controls $(\mathrm{p}=0,001)$. When mean PON1 and PONAryl levels of the patients diagnosed with simple febrile 
convulsion and the healthy controls were compared, no statistically significant difference was determined, although they were lower in patients with febrile convulsion $(p>0,05)$. When mean serum PON1 levels of the patients using levetiracetam and those using sodium valproate were compared, PON1 levels were determined to be statistically significantly lower in those using sodium valproate $(\mathrm{p}=0,005)$. No statistically significant difference was determined in serum PONAryl levels, although it was lower in those using sodium valproate $(p>0,05)$ (Table 4$)$. When mean serum PON1 and PONAryl levels of the patients using levetiracetam and the patients diagnosed with febrile convulsion were compared, no statistically significant difference was determined, although they were lower in those using levetiracetam ( $p>0,05)$. When mean serum PON1 levels of the patients using sodium valproate and the patients diagnosed with febrile convulsion were compared, PON1 levels were determined to be statistically significantly lower in those using sodium valproate $(\mathrm{p}=0,005)$. Serum PONAryl levels also were determined to be statistically significantly lower in those using sodium valproate $(\mathrm{p}=0,033)$.

Table 1: Distribution of the patients and control group by age and gender.

\begin{tabular}{|c|c|c|c|c|c|}
\hline & $\begin{array}{l}\text { Patients using Sodium } \\
\text { Valproate }(\mathrm{n}=30)\end{array}$ & $\begin{array}{l}\text { Patients using Levetiracetam } \\
\qquad(\mathrm{n}=30)\end{array}$ & $\begin{array}{l}\text { Patients with Febrile Convulsion } \\
\qquad(\mathrm{n}=30)\end{array}$ & $\begin{array}{l}\text { Healthy Controls } \\
\qquad(\mathrm{n}=30)\end{array}$ & Total $(n=120)$ \\
\hline Age & $3,65 \pm 0,83$ & $4,01 \pm 0,83$ & $3,69 \pm 0,81$ & $3,76 \pm 0,82$ & $3,78 \pm 0,82$ \\
\hline Female & 15 & 16 & 17 & 16 & 64 \\
\hline Male & 15 & 14 & 13 & 14 & 56 \\
\hline
\end{tabular}

Table 2: Comparison of serum Paraoxonase 1 and Arylesterase levels of patient and control groups.

\begin{tabular}{|c|c|c|c|}
\hline & Patients (n=90) & Healthy Controls (n=30) & p \\
\hline Paraoxonase 1 (U/L) & $204,84 \pm 74,67$ & $278,41 \pm 87,45$ & 0,001 \\
\hline Arylesterase (kU/L) & $1070,43 \pm 249,11$ & $1250,65 \pm 226,06$ & 0,001 \\
\hline
\end{tabular}

Table 3: Comparison of Paraoxonase 1 and Arylesterase levels between groups.

\begin{tabular}{|c|c|c|c|c|c|}
\hline & $\begin{array}{l}\text { Patients using Sodium } \\
\text { Valproate }(\mathrm{n}=30)\end{array}$ & $\begin{array}{l}\text { Patients using Levetiracetam } \\
\qquad(n=30)\end{array}$ & $\begin{array}{l}\text { Patients with Febrile } \\
\text { Convulsion }(n=30)\end{array}$ & Healthy Controls $(n=30)$ & $\mathbf{p}$ \\
\hline Paraoxonase $1(\mathrm{U} / \mathrm{L})$ & $170,63 \pm 59,38$ & $216,34 \pm 63,23$ & $227,56 \pm 87,78$ & $278,41 \pm 87,45$ & 0,001 \\
\hline Arylesterase (kU/L) & $1000,95 \pm 135,01$ & $1068,36 \pm 235,65$ & $1141,98 \pm 326,19$ & $1250,65 \pm 226,06$ & 0,001 \\
\hline
\end{tabular}

Table 4: Comparison of Paraoxonase 1 and Arylesterase levels by the drugs used.

\begin{tabular}{|c|c|c|c|}
\hline & Patients using Sodium Valproate (n=30) & Patients using Levetiracetam (n=30) & $\mathbf{p}$ \\
\hline Paraoxonase 1 (U/L) & $170,63 \pm 59,38$ & $216,34 \pm 63,23$ & 0,005 \\
\hline Arylesterase (kU/L) & $1000,95 \pm 135,01$ & $1068,36 \pm 235,65$ & $>0,05$ \\
\hline
\end{tabular}

\section{Discussion}

In pathophysiology of severe neurological disorders such as epileptic seizures, stroke, neurodegenerative disorders and neurotrauma, oxidative stress that emerges as a result of impairment of the balance between oxidants and antioxidants plays a role [11]. Lipid peroxidation, which leads to destruction of cell membrane, is the most important effect of oxidative stress. Susceptibility of the brain to oxidative damage is high due to high metabolic demand, high polyunsaturated fatty acid content, insufficient repair capacity and high iron load [12]. In vivo detection of free radicals is difficult because of their short lifespan; therefore, markers of lipid peroxidation are used as an indicator of oxidative stress in clinical and experimental studies [13]. It is known that oxidant-antioxidant balance is impaired in patients with febrile seizure and epilepsy via increased lipid peroxidation and occurrence of neuronal cell damage after seizures. In this study, it was determined that valproate had a potent inhibition effect and levetiracetam had weak inhibition effect on PON1 and PONAryl activities, and it was concluded that these patients with impaired oxidative balance should be followedup closely due to increased risk of atherosclerosis. Although it is well-known that epileptic seizures cause oxidative stress, effect of febrile seizures on oxidative stress is unclear [14]. Seizures trigger various biochemical processes, including extracellular calcium flow, activation of membrane phospholipases and release of free fatty acids, eicosanoids, lipid peroxides and free radicals [15]. In a study on effect of increments in body temperature on oxidative stress which was conducted by[16], level of malonyl-aldehyde, which has a protective effect on temperature-mediated oxidative stress, was determined to be increased and it was shown that antioxidant enzymes and their metabolytes may play an important role in protection of cells against temperature stress. In the study conducted by Akarsu et al. [17]. however, it was observed that both fever and convulsions influenced the oxidative mechanism, and while fever did not increase oxidative stress in healthy controls, higher levels were determined in patients having a febrile seizure compared to the healthy control group. In the study conducted by 
Gunes et al. [8]. it was concluded that oxidant-antioxidant balance was impaired in children having a febrile seizure, and neuronal cell injury may occur after febrile seizures via increased lipid peroxidation and alterations in levels of antioxidant enzymes. In our study, when mean PON1 and PONAryl levels of the patients diagnosed with simple febrile convulsion and the healthy controls were compared, no statistically significant difference was determined, although they were lower in patients with febrile convulsion. These results suggest that oxidant-antioxidant balance is impaired in children having a febrile seizure via increased lipid peroxidation and occurrence of neuronal cell injury after febrile seizures Figures 1 \& 2 .

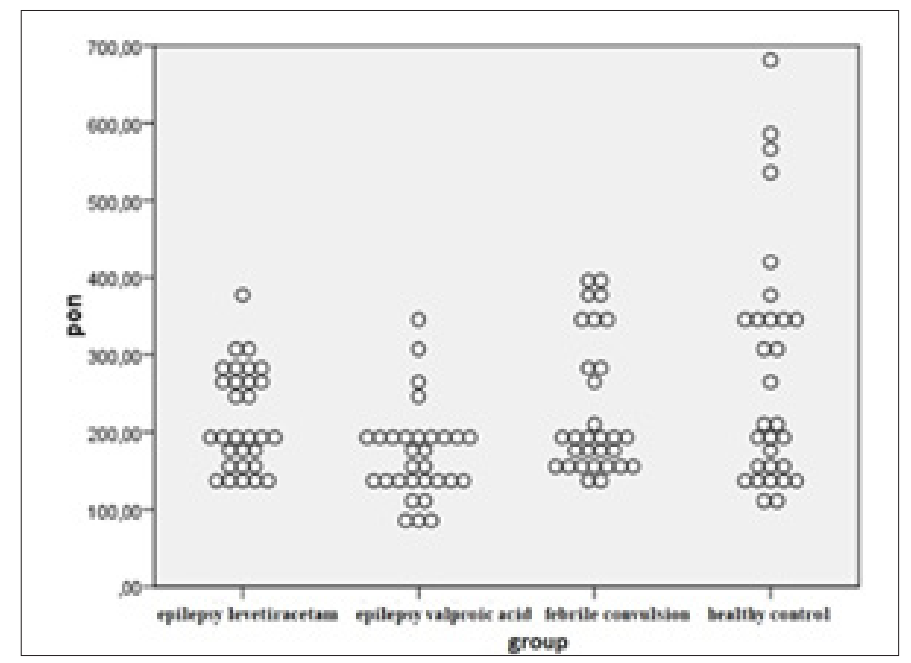

Figure 1: Distribution of Paraoxonase 1 (U/L) levels by group.

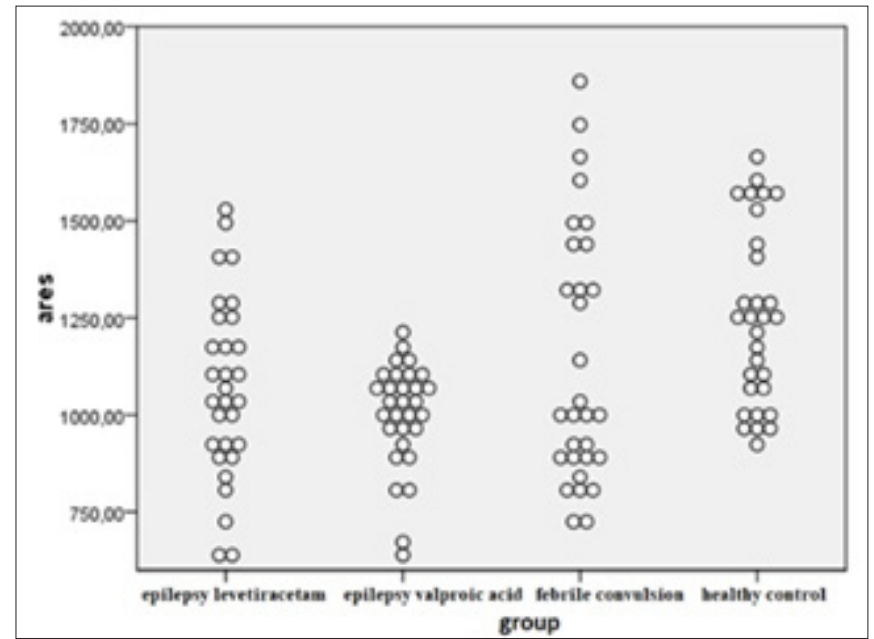

Figure 2: Distribution of Aryl esterase (kU/L) levels by group.

Previous studies suggest that anti-epileptic drugs trigger or aggravate oxidative injury in epileptic patients [18]. Drugs used for various epileptic syndromes such as valproic acid, carbamazepine, phenytoin or phenobarbital increase lipid peroxidation and nucleic acid oxidation, altering the antioxidant defense system $[19,20]$. Again, many studies have demonstrated that anti-epileptic drug therapy may lead to an increase in oxidant markers [21]. In the study conducted by Chen et al. [22]. decreased activity of serum PON1 compared to the control group was determined in patients receiving levetiracetam treatment and it was concluded that levetiracetam reduced serum PON1 activity and increased oxidized LDL in epilepsy patients. In the study conducted by Beydemir et al. [23], however, it was shown that all antiepileptic drugs inhibited serum PON1 but levetiracetam had weak inhibition effect on PON1 activity. In the study which Karikas et al. [24] conducted on epilepsy patients using sodium valproate, it was concluded that reduction of PON1 and PONAryl activities depended not only on direct effect of the drug on anti-atherogenic enzymes, but also on the oxidative stress caused by sodium valproate. Also in our study, it was determined consistently with the literature that PON1 and PONAryl activities were statistically significantly decreased in epilepsy patients using sodium valproate, were higher in epilepsy patients using levetiracetam compared to those using sodium valproate, although they were decreased compared to the healthy controls. This, hence, is such as to support the view that levetiracetam has a weak inhibition effect on PON1 and PONAryl activities. There are 
different studies on increased risk of atherosclerosis in children and adults receiving antiepileptic treatment [25]. In the study conducted by Ruperez et al. [26], no difference was determined in PON1 levels between obese and non-obese children but it was found to be associated with markers involved in the lipid metabolism such as lactonase activity, HDL and apolipoprotein-A1. In the study conducted by Chistiakov et al. [27], it was concluded that PONs had athero-protective properties including antioxidant activity, anti-inflammatory effect, preservation of HDL function, stimulation of cholesterol flow, anti-apoptosis, anti-thrombosis and anti-adhesion, and contributed to susceptibility to and prevention of atherosclerosis-related diseases. There are also studies showing that high serum PON1 activity destructs pro-inflammatory molecules that are involved at the initiation and progression of the atherosclerotic lesions, reducing risk of coronary artery disease [28]. In our study, patients with abnormal liver function tests and lipid profile and obese patients were not included; determination of serum PON1 and PONAryl levels to be low in these patients using levetiracetam and sodium valproate despite of lack of an obvious risk of atherosclerosis suggests that these patients should be followed-up closely due to increased risk of atherosclerosis and, additionally, PON1 and PONAryl may be used as early markers for risk of atherosclerosis. In our study in which effects of antiepileptic drugs on PON1 and PONAryl activities in children with epilepsy and simple febrile convulsion were investigated, it was concluded that oxidant-antioxidant balance is impaired in patients with febrile seizure and epilepsy via increased lipid peroxidation and occurrence of neuronal cell damage after seizures, sodium valproate had a potent inhibition effect and levetiracetam had weak inhibition effect on PON1 and PONAryl activities. Although determination of serum PON1 and PONAryl levels to be low in these patients using levetiracetam and sodium valproate despite of lack of an obvious risk of atherosclerosis suggests that these patients should be followed-up closely due to increased risk of atherosclerosis, further studies involving longer patient follow-up durations are necessary in order to determine whether these markers can be used as early markers for the risk. In conclusion, Understanding the oxidative stress, which is influenced by several causes such as age, lifestyle, metabolic disorders, toxic chemicals or drugs, may shed light on emergence of new therapeutically approaches for treatment of epilepsy and febrile convulsion and for pathophysiology of epilepsy.

\section{References}

1. Scheffer IE, Berkovic S, Capovilla G (2017) ILAE classification of the epilepsies: position paper of the ILAE Commission for classification and terminology. Epilepsia 58(4): 512-521.

2. Aaberg KM, Gunnes N, Bakken IJ (2017) Incidence and prevalence of childhood epilepsy: A nationwide cohort study. Pediatrics 139(5): e20163908.

3. Rosman NP (2003) Evaluation of the child who convulses with fever. Paediatr Drugs 5: 457-461.

4. Perrone S, Negro S, Tataranno ML, Buonocore G (2010) Oxidative stress and antioxidant strategies in newborns. J Matern Fetal Neonatal Med 23(3): 63-65.
5. Menon B, Ramalingam K, Kumar RV (2012) Oxidative stress in patients with epilepsy is independent of antiepileptic drugs. Seizure. 21(10): 780-784.

6. Devi PU, Manocha A, Vohora D (2008) Seizures, antiepileptics, antioxidants and oxidative stress: an insight for researchers. Expert Opin Pharmacother 9(18): 3169-3177.

7. Nazıroğlu M, Yürekli VA (2013) Effects of antiepileptic drugs on antioxidant and oxidant molecular pathways: focus on trace elements. Cell Mol Neurobiol 33(5): 589-599.

8. Güneş S, Dirik E, Yiș U (2009) Oxidant status in children after febrile seizures. Pediatr Neurol 40(1): 47-49.

9. Furlong CE, Marsillach J, JarvikP, Costa LG (2016) Paraoxonases-1, -2 and 3: What are their functions? Chem Biol Interact 25: 51-62.

10. Ikeda Y, Suehiro T, Itahara T (2007) Human serum paraoxonase concentration predicts cardiovascular mortality in hemodialysis patients. Clinical nephrology 67(6): 358-365.

11. Valko M, Leibfritz D, Moncol J, Cronin MT, Mazur M, et al. (2007) Free radicals and antioxidants in normal physiological functions and human disease. Int J Biochem Cell Biol 39(1): 44-84.

12. Beal MF (1998) Mitochondrial dysfunction in neurodegenerative diseases. Biochim Biophys Acta 1366(1): 211-223.

13. Halliwell B (1992) Reactive oxygen species and the central nervous system. J Neurochem 59(5): 1609-1623.

14. Patel MN (2002) Oxidative stress, mitochondrial dysfunction, and epilepsy. Free Radic Res 36(11): 1139-1146.

15. Eraković V, Zupan G, Varljen J, Radošević S, Simonić A (2000) Electroconvulsive shock in rats: changes in superoxide dismutase and glutathione peroxidase activity. Brain Res Mol Brain Res 76(2): 266-274.

16. Ali MB, Hahn EJ, Paek KY (2005) Effects of temperature on oxidative stress defense systems, lipid peroxidation and lipoxygenase activity in Phalaenopsis. Plant Physiol Biochem 43(3): 213-223.

17. Akarsu S, Yilmaz S, Ozan S, Kurt A, Benzer F, et al. (2007) Effects of febrile and afebrile seizures on oxidant state in children. Pediatr Neurol 36(5): 307-311.

18. Varoglu AO, Yildirim A, Aygul R, Gundogdu OL, Sahin YN (2010) Effects of valproate, carbamazepine, and levetiracetam on the antioxidant and oxidant systems in epileptic patients and their clinical importance. Clin Neuropharmacol 33(3): 155-157.

19. Yüksel A, Cengiz M, Seven M, Ulutin T (2000) Erythrocyte glutathione, glutathione peroxidase, superoxide dismutase and serum lipid peroxidation in epileptic children with valproate and carbamazepine monotherapy. J Basic Clin Physiol Pharmacol 11(1): 73-81.

20. Hamed SA, Abdellah MM, Melegy N (2004) Blood levels of trace elements, electrolytes, and oxidative stress/antioxidant systems in epileptic patients. J Pharmacol Sci 96(4): 465-473.

21. Jakubus T, Michalska JM, Lukawski K, Janowska A, Czuczwar SJ (2009) Atherosclerotic risk among children taking antiepileptic drugs. Pharmacol Rep 61(3): 411-423.

22. Chen W, Tan Y, Ge Y, Chen Y, Liu X (2015) The effects of levetiracetam on cerebrospinal fluid and plasma npy and gal, and on the components of stress response system, hs-crp, and s100b protein in serum of patients with refractory epilepsy. Cell Biochem Biophys 73(2): 489-494.

23. Beydemir Ş, Demir Y (2017) Antiepileptic drugs: Impacts on human serum paraoxonase-1. J Biochem Mol Toxicol 31(6).

24. Karikas GA, Schulpis KH, Bartzeliotou A (2009) Early effects of sodium valproate monotherapy on serum paraoxonase/arylesterase activities. Scand J Clin Lab Invest 69(1): 31-35. 
25. Belcastro V, Egidio C, Striano P, Verrotti A (2013) Metabolic and endocrine effects of valproic acid chronic treatment. Epilepsy Res 107(1-2): 1-8.

26. Rupérez AI, López GO, Gil F (2013) Paraoxonase 1 activities and genetic variation in childhood obesity. Br J Nutr 110: 1639-1647.

27. Chistiakov DA, Melnichenko AA, Orekhov AN, Bobryshev YV (2017)
Paraoxonase and atherosclerosis-related cardiovascular diseases. Biochimie 132: 19-27.

28. Ferré N, Camps J, Prats E (2002) Serum paraoxonase activity: a new additional test for the improved evaluation of chronic liver damage. Clin Chem 48(2): 261-268.

For possible submissions Click below: 